\title{
EL NUEVO MODELO PRODUCTIVO CHINO Y EL PLAN MADE IN CHINA 2025 AMENAZADO POR LA PANDEMIA DEL COVID-19. ANÁLISIS COMPARADO DESDE 2014 HASTA LA ACTUALIDAD
}

THE NEW CHINESE PRODUCTION MODEL AND THE MADE IN CHINA 2025 PLAN THREATENED BY THE COVID-19 PANDEMIC. COMPARATIVE ANALYSIS FROM 2014 TO PRESENT

Mar Perona Alfageme

Profesor Asociado Grado en Administración y Dirección de Empresas.

Universidad Complutense, (España).

E-mail: mmperona@ucm.es ORCID: https://orcid.org/0000-0003-3823-2133

Fernando Martínez-López

Profesor, Grado en Administración y Dirección de Empresas y Grado en Dirección Comercial y Marketing. Departamento de Estudios Sociales, Universidad Alfonso X el Sabio, (España). E-mail: fmartlpe@uax.es ORCID: https://orcid.org/0000-0001-6490-0997

\section{Citación sugerida:}

Perona, M. P., y Martínez-López, F. (2020). El nuevo modelo productivo chino y el plan Made in China 2025 amenazado por la pandemia del COVID-19. Análisis comparado desde 2014 hasta la actualidad. 3C Empresa. Investigación y pensamiento crítico. Edición Especial COVID-19: Empresa, China y Geopolitica, 29-51. https://doi.org/10.17993/3cemp.2020.edicionespecial1.29-51 


\section{RESUMEN}

Este trabajo analiza el proceso de transformación económica que está experimentando China desde del año 2014 cuando se desarrollan un conjunto de planes para corregir el rumbo de la economía. Este conjunto de ambiciosas medidas articuladas en amplio horizonte temporal están orientadas a reinventar su modelo productivo de bajo coste hacia la producción de bienes y servicios de mayor valor añadido (Plan Made in China 2025). A compensar la brecha existente en el desarrollo regional (Proyecto de la Nueva Ruta de la Seda) y a erradicar la pobreza del país que desde 1980 ha sacado a casi mil millones de personas de la pobreza.

Se presenta un análisis crítico comparado de la efectividad de estas mejoras, se realiza un análisis particular sobre el desarrollo del comercio electrónico y se plantean unas reflexiones sobre el impacto que la pandemia COVID-19 ha tenido sobre ellas. Se tiende la vista al futuro para concluir que a lo largo del S. XXI Asia en general y china en particular tendrán un papel protagonista en el desarrollo económico mundial.

\section{PALABRAS CLAVE}

China, Innovación, Comercio electrónico, COVID-19, Transformación económica, Plan Made in China 2025. 


\section{ABSTRACT}

This paper analyses the process of economic transformation experienced in China since 2014, when a set of plans were developed to correct the course of the economy. This set of ambitious measures articulated over a wide timeframe are aimed at re-inventing its low-cost production model towards the production of high added value goods and services (Plan Made in China 2025). To close the existing gap in the regional development (New Silk Road Project) and to eradicate poverty in a country that since has lifted almost a billion people out of poverty. A critical analysis of the effectiveness of these measures is presented, a particular analysis is carried out on the development of e-commerce and some reflections are made on the impact of the COVID-19 pandemic. Looking at the future, this paper concludes that throughout the XXI century Asia in general and China will have a leading role in the world economic development.

\section{KEYWORDS}

China, Innovation, Electronic commerce, COVID-19, Economic transformation, Plan Made in China 2025. 


\section{INTRODUCCIÓN}

Durante las últimas cuatro décadas China ha experimentado un proceso de transformación único en el mundo. Como consecuencia, su PIB se ha multiplicado por 65 y casi mil millones de personas han logrado traspasar del umbral internacional de pobreza marcado por el Banco Mundial. Este proceso de transformación arranca a mediados de la década de los 70 con la adopción de un conjunto de políticas liberales que convierten a China en "la fábrica del mundo" especializada en la producción a bajo coste.

A lo largo de la segunda década de los 2000 después de 30 años de crecimiento desbordado, este modelo muestra signos de agotamiento. En el Tercer Pleno del XVIII Congreso del Partido Comunista celebrado en el año 2013, se adoptan una serie de medidas con el objetivo de enderezar el rumbo de la economía corrigiendo los desequilibrios existentes y de transformar el modelo productivo hacia una producción de mayor valor añadido e innovación. En este sendero de transformación, irrumpe la pandemia del COVID-19 en diciembre de 2019.

Si bien a la fecha de cierre de este trabajo no es sencillo cuantificar sus efectos en la economía, según proyecciones del Fondo Monetario Internacional, el PIB de China va a crecer a un $1 \%$ en 2020 y un 8,2\% en 2021. Este dato supone una contracción de su PIB en el primer cuatrimestre de 2020 de un 6,8\% respecto al mismo periodo de 2019 (China Dashboard, 2020). Para poner este dato en perspectiva, este organismo estima que el PIB mundial va a decrecer en 2020 en un 4,9\% y los países de la zona euro lo harán en un 10,2\% (FMI, 2020).

Este trabajo se centra en la situación China a partir de 2014. Presenta y analiza los principales problemas que amenazan a su economía y analiza las medidas que se están tomando. Se hará especial énfasis en el desarrollo del comercio electrónico y su relación con la innovación en las empresas chinas. Introduce el efecto que la pandemia del COVID-19 ha tenido tanto a nivel económico como social en esta senda de crecimiento.

En concreto se van a tratar los siguientes aspectos: la lucha por la eliminación de la pobreza y los desequilibrios regionales, el Plan de inversiones enmarcada en la Nueva Ruta de la Seda y el diseño del nuevo modelo productivo con el Plan Made in China 2025. 


\section{OBJETIVOS Y METODOLOGÍA}

El objetivo principal de este trabajo consiste en ofrecer una visión evolutiva y comparada del desarrollo económico de China desde 2014 hasta la actualidad.

Como objetivos secundarios se plantean los siguientes:

- Estudiar el desarrollo del comercio electrónico como una de las principales palancas de los planes de innovación de China.

- Analizar el impacto que está teniendo la pandemia del COVID-19 en el desarrollo económico chino.

Para alcanzar estos objetivos, se ha elegido el método comparativo en contextos internacionales como herramienta de análisis. Esta metodología científica como indican Sartori y Morlino (1994) se puede aplicar para realizar observaciones sistemáticas de dos o más entidades macrosociales o para diferentes momentos temporales de una misma sociedad. En particular, Wolff y Resnick (2012) manifiestan la importancia de utilizar este método en Economía para evitar centrarse en un solo paradigma.

\section{DESARROLLO}

A lo largo de este apartado se revisarán tres ejes que articulan la estrategia económica china encaminada a reorientar la economía y a situarla en un rumbo sostenido de crecimiento a medio plazo. En primer lugar, se abordará los esfuerzos para la eliminación de la pobreza, a continuación, la eliminación de los desequilibrios regionales que han existido en cuanto al desarrollo económico y la revitalización de la antigua ruta de la Seda (Proyecto One Belt One Road) como mecanismo para compensar estos desequilibrios. En tercer lugar, el estímulo a la innovación en el modelo productivo chino articulada mediante el Plan "Made in China 2025". Dentro de dicho plan uno de los aspectos relevantes es potenciar el comercio electrónico, a ese aspecto se dedicará el último apartado de esta sección.

\subsection{LUCHA POR LA ELIMINACION DE LA POBREZA}

La drástica reducción de la pobreza es uno de los hechos más relevantes para China hoy: según datos del Banco Mundial, desde que se pusieron en marcha las reformas, cerca de 800 millones de personas han salido de la pobreza en China. Ningún otro país ha logrado 
un nivel de reducción similar en tan poco tiempo. Existe una suerte de "contrato social" entre los ciudadanos y el Gobierno caracterizado porque los primeros ceden algo de sus derechos a cambio de riqueza y estabilidad económica (Ryder, 2017).

El Gobierno se ha comprometido a continuar esta tarea con el objetivo de erradicar la pobreza rural en el año 2020. En el año 2016 tan solo un 0,5\% de la población vivía bajo el umbral internacional de la pobreza, lo que suponen casi 7 millones de habitantes, considerado por esa institución como 1,9 USD al día en una paridad de poder de compra (PPA) y a precios de 2011 (véase Figura 1).

El Banco Mundial estima que como consecuencia del COVID-19 va a producirse el primer aumento de la pobreza mundial desde la crisis financiera de 1998. Con mucha cautela a causa de la incertidumbre de la situación, se estima que el número de pobres extremos en el mundo se verá incrementado entre 71 y 100 millones. De esta cifra, casi la mitad de los "nuevos pobres" estarán en el Sur de Asia, siendo India uno de los países más afectados. Son precisamente los hogares con niveles de ingresos más bajos los que más van a acusar los efectos del COVID-19 lo que va a suponer un retroceso en los avances (FMI, 2020). Para China, se estima que alrededor de un millón de personas volverán a niveles de extrema pobreza en 2020. Este dato, por lo tanto, dificulta el objetivo del Gobierno de eliminar la pobreza rural por completo en el año 2020.

Muy relacionado con este tema, los importantes desequilibrios regionales son otro de los problemas que atenazan a la sociedad china. Trataremos este tema en el siguiente punto.

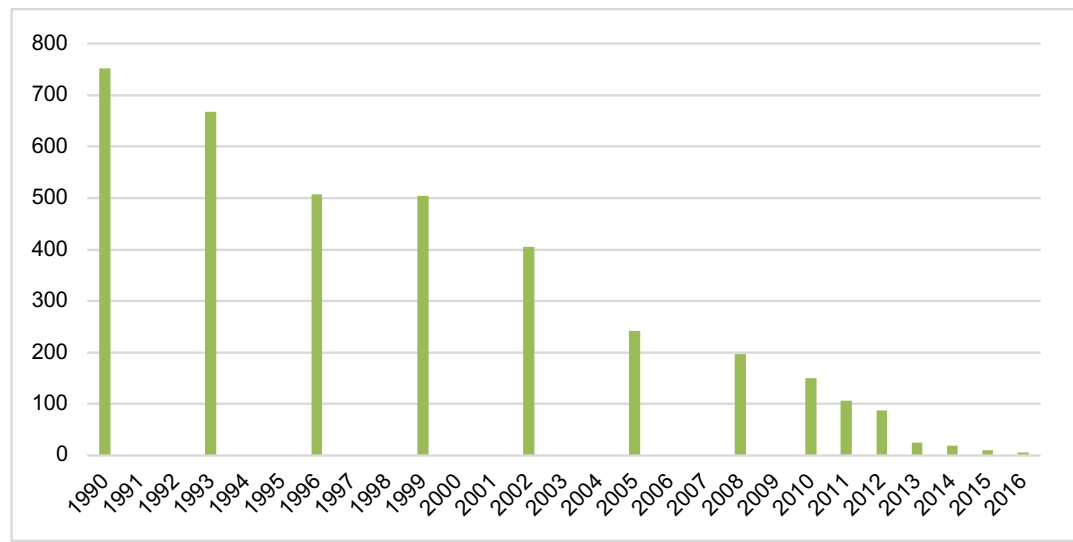

Figura 1. Personas que viven en China bajo el umbral internacional de pobreza (en millones de personas).

Fuente: elaboración propia con datos del Banco Mundial (2020). 


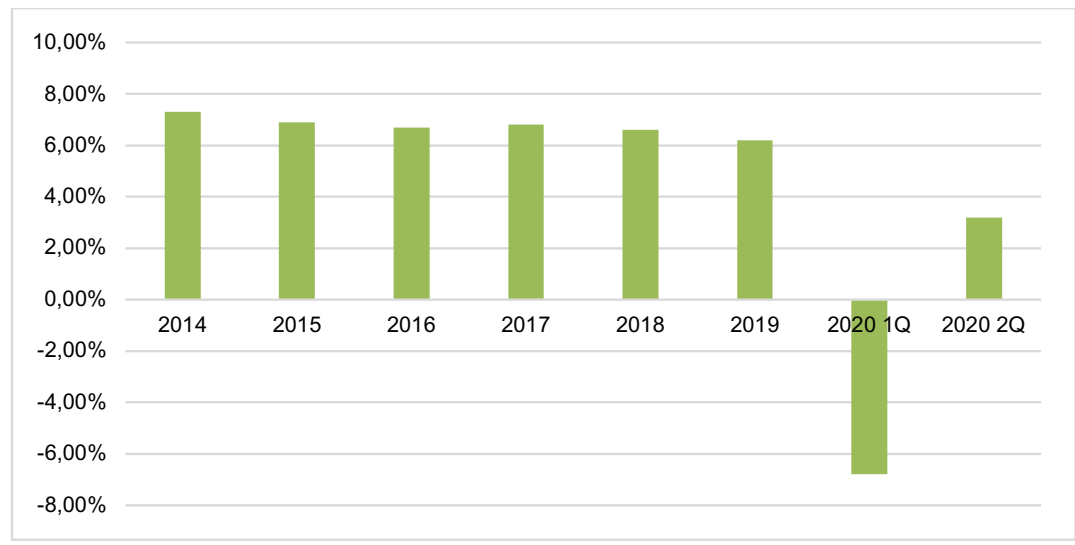

Figura 2. Variación interanual del PIB en China (en porcentaje).

Fuente: elaboración propia.

\subsection{CORRECCION DE LOS DESEQUILIBRIOS REGIONALES: LA NUEVA RUTA DE LA SEDA}

Existe en China una clara brecha en cuanto a los niveles de desarrollo económico regional. Las grandes ciudades y las regiones costeras orientales, bañadas por el Mar de China son las áreas más desarrolladas. Este es un patrón de desarrollo común en muchos países del mundo, en los que la proximidad al mar genera mayor accesibilidad y menores costes de transporte. Además de la proximidad al mar, la geografía del país contribuye en gran parte a esta brecha ya que la orografía dificulta la conexión entre la mitad oriental y la mitad occidental del país, asentada en numerosas cadenas montañosas y desiertos.

Otra causa de los desequilibrios regionales son las políticas económicas puestas en marcha desde finales de la década de los 70: es el denominado "periodo de reforma y apertura económica" liderado por Deng Xiaoping que rompe con la política aislacionista de la época de Mao Zedong.

Estas reformas pusieron el foco en el desarrollo de estas zonas costeras bañadas por el Mar de China y las abrieron a la inversión extranjera y al comercio exterior. Como consecuencia se agrandó la brecha entre las regiones orientales costeras y las interiores occidentales. Brecha que todavía persiste como se puede observar en la Tabla 1.

En esta tabla se puede ver el PIB per cápita por provincia en China en el año 2018 expresada en Yuanes. Aparecen reflejadas las 10 primeras y las 10 últimas provincias en términos de 
su PIB. También aparece la media nacional. De las 10 primeras todas están en la costa a excepción de Pekín, de Mongolia interna y de Hubei. Las provincias con un PIB más bajo se sitúan en el sur oeste del país en las estribaciones del Himalaya.

Tabla 1. PIB per cápita por provincias en 2018 expresado en yuanes.

\begin{tabular}{|c|c|}
\hline Provincia & $\begin{array}{c}\text { PIB per capita 2018 } \\
\text { (expresado en yuanes) }\end{array}$ \\
\hline Pekín & 140211 \\
\hline Shanghai & 134982 \\
\hline Tianjing & 120711 \\
\hline Jiangsu & 115168 \\
\hline Zhejiang & 98643 \\
\hline Fujiang & 91197 \\
\hline Guangdong & 86412 \\
\hline Shandong & 76267 \\
\hline Mongolia interna & 68302 \\
\hline Hubei & 66616 \\
\hline Media nacional & 64644 \\
\hline Anhui & 47712 \\
\hline Qinghai & 47689 \\
\hline Jiangxi & 47434 \\
\hline Shanxi & 45328 \\
\hline Tibet & 43398 \\
\hline Heilongjang & 43274 \\
\hline Guangxi & 41489 \\
\hline Guizhou & 41244 \\
\hline Yunnan & 37136 \\
\hline Gansu & 31336 \\
\hline & \\
\hline & 0 \\
\hline
\end{tabular}

Fuente: (Textor, 2020).

El programa más ambicioso en este contexto es la revitalización de las antiguas rutas comerciales que constituían la histórica "Ruta de la Seda" que, con inversiones millonarias, conectan China con Occidente a través de diversas rutas por tierra y por mar. Para este proyecto, denominado por los chinos Una Franja una Ruta (One Belt One Road en inglés) el Gobierno Chino ha dotado un presupuesto de 3 trillones de USD para invertir en las próximas décadas. Este dinero se canaliza en forma de préstamos para la construcción de infraestructuras. Con este proyecto el Gobierno pretende revitalizar las conexiones comerciales de las recónditas provincias del centro y noroeste tanto hacia oriente como 
hacia occidente, activar la capacidad productiva infrautilizada y fomentar la prosperidad y el desarrollo económico de las regiones menos favorecidas del país.

A pesar de la irrupción de la pandemia COVID-19 este proyecto va a continuar siendo una prioridad para el Gobierno Chino. Si bien a corto plazo, se está produciendo un desvío de la atención y de los recursos económicos hacia otros fines socio sanitarios y financieros, por otro lado, está cediendo protagonismo a la iniciativa privada como motor inversor (Boo, David y Simfpendorfer, 2020).

Se está reforzando el interés en la zona del sudeste asiático como destino de la inversión china (Vietnam, Camboya, Laos): los menores costes salariales y la relativa estabilidad de esta zona son el atractivo que ofrece esta zona a la inversión privada china. También el sector público está realizando inversiones en infraestructuras (puertos, carreteras...) para dar apoyo a la inversión privada china. Otros importantes beneficiarios son Pakistán, Bangladesh y Myanmar en parte debido a su proximidad con china y al aumento de potencial de su mercado.

Por sectores, las industrias que originariamente acapararon más atención han sido infraestructura y energía si bien en la actualidad se aprecia cómo el interés se está ampliando a industrias digitales como el comercio e internet (Deloitte, 2018). Precisamente a industrias que se están viendo favorecidas como consecuencia de la pandemia. Destacan las aplicaciones médicas de inteligencia artificial como los códigos $Q R$ para identificar y seguir la pista a los portadores del virus, las plataformas digitales para la consulta médica y los sistemas para la gestión de la información sanitaria (Boo et al., 2020). Por otro lado, las redes de comunicación, de compañías ya establecidas en la ruta de la seda encontrarán oportunidades, igualmente, destaca el protagonismo del comercio electrónico que se comentará más adelante.

\subsection{DISEÑO DE UN NUEVO MODELO PRODUCTIVO Y PLAN MADE IN CHINA 2025}

El modelo económico que ha sustentado tantos años de desarrollo y bonanza y que ha sacado a tantos millones de personas de la pobreza se encuentra agotado. Por ello, se pretende abandonar el enfoque que hasta el momento ha predominado, basado en la 
producción de bajo coste para centrarse en la innovación y en la producción de bienes y servicios de alto nivel añadido que sean competitivos a nivel internacional. El objetivo es pasar de la producción "hecha en China" a la "inventada en China". Además, este cambio tiene que ir a la par con una retirada de la inversión pública, dejando paso al consumo privado como motor de la economía.

El modelo se ha agotado porque China ya no es competitiva como productora líder en costes. El desarrollo económico experimentado, ha propiciado un aumento de los salarios, erosionando la competitividad de su economía. Siguiendo datos de Statista (2020), desde el año 2010 la senda de crecimiento anual de los salarios llega casi al 10\% anual, totalizando un incremento de un 122\% entre 2010 y 2018. Esta fuerte subida de los costes laborales ha impulsado el desplazamiento de muchas industrias intensivas en mano de obra hacia otros países del sudeste asiático, con unos salarios que pueden llegar a ser la quinta parte de los de China.

Para frenar el deterioro en su productividad, China está enfocando la producción hacia sectores de mayor valor añadido como hicieron en su tiempo Japón y los países denominados "tigres asiáticos" evitando caer en la "trampa de la renta media".

Un aliciente para este cambio en el modelo productivo viene de la voluminosa clase media china, que está aumentando su calidad de vida material y que demanda cada vez más, bienes y servicios de mayor calidad y valor añadido (recordemos los cientos de millones de personas que han salido de la pobreza). Las empresas chinas tienen un inmenso mercado por atender.

Desde una perspectiva estratégica la independencia tecnológica respecto a Occidente es una prioridad. Recientemente se ha puesto en marcha el "Plan Made in China 2025" que incluye una serie de acciones encaminadas a situar al país a la vanguardia del desarrollo científico y tecnológico mundial. Como muchos de los planes en China, se trata de una estrategia a medio plazo claramente diseñada y articulada en tres fases: La primera que se extiende hasta 2025 se centra en la reducción de las diferencias con los países más avanzados, en la segunda fase (2035) el objetivo es fortalecer la posición para en la tercera fase (2045) lograr posicionarse a la vanguardia de la innovación mundial. Una vez más, vemos la capacidad de planificación y ejecución a medio y largo plazo lo que confiere a China una enorme ventaja frente al enfoque cortoplacista de las potencias occidentales. 
El plan contempla la industria más puntera pero también la más tradicional. Se apoya en la automatización y la producción sostenible y se focaliza en una serie de sectores económicos estratégicos entre los que se encuentra las nuevas energías, las telecomunicaciones, el equipamiento médico o las herramientas de control numérico y la robótica.

La inversión en I+D se va a potenciar hasta alcanzar en 2020 en un 2,5\% del PIB. Para 2020 se espera que China supere a Estados Unidos como inversor en I+D. Esta inversión se materializará en la creación de 20 centros nacionales de I+D en 2020 y 40 más para el año 2025 (ICEX, 2016).

La formación y la atracción de talento son otro elemento de este plan. Se está mejorando la eficiencia del sistema educativo tanto universitario como profesional, y paralelamente se está desarrollando un intenso programa de repatriación del talento chino ubicado en el extranjero, que hasta la fecha a traído a casa a cerca de 2,5 millones de chinos (Bregolat, 2018).

Otro de los objetivos que contempla este plan es el apoyo al desarrollo del mercado de capitales, habida cuenta de la escasa experiencia que acumulan los mercados de capitales chinos y su sistema financiero como canalizadores de fondos para inversiones de riesgo (Lieberthal, 2011).

Este esfuerzo innovador ya está dando sus frutos en un tiempo récord y el país se está dotando de un tejido empresarial en algunos casos de primera línea. Es el caso de empresas como Tencent, que es la propietaria de WeChat, de Baidú o de la compañía fabricante de teléfonos Xiaomi.

Si bien el programa declara la mayor apertura del sector manufacturero a la inversión extranjera, existe preocupación por parte de las empresas multinacionales basadas en China sobre cierto proteccionismo en las medidas e incentivos para los productores locales:

Muchas empresas que se quieren instalar a operar en el gigante asiático han experimentado dificultades legales o de acceso a los mercados (Blanco, 2019). Grandes empresas como Facebook o Amazon tienen vetada o muy limitada su actuación en China. Esto permite que las empresas locales se desarrollen en un régimen de monopolio u oligopolio y se beneficien del tamaño de su mercado doméstico explotando inmensas economías de escala. 
La Cámara de comercio de Europa en China (2018) señala que este proteccionismo puede resultar a la larga contraproducente: $\mathrm{Al}$ estar las grandes compañías chinas al abrigo de la competencia internacional, se impide el desarrollo competencias estratégicas necesarias para ser competitivas en el extranjero.

Además de las reformas que se han comentado destinadas a la oferta, también se contempla otro paquete orientado a la demanda. El nuevo modelo chino tiene como objetivo desplazar el motor del crecimiento de la economía que durante estos años ha sido la inversión pública y las exportaciones, a un modelo centrado en el consumo y la inversión privadas. Se han puesto en marcha una serie de medidas para aumentar la demanda interna y potenciar el papel de las empresas privadas. La realidad es que la demanda interna se está ralentizando y el consumo interno está estancado. Como se ha mostrado en la figura 2, el PIB está creciendo a un ritmo anual más lento de las tres últimas décadas y en los tres primeros meses de 2020 la incidencia de la pandemia se ha cuantificado con una contracción del PIB de más de un $6 \%$.

En el contexto internacional la guerra comercial con los Estados Unidos, abierta el 2018, incide en el debilitamiento de la ya delicada demanda interna. Algunos economistas cifran el efecto de la guerra comercial entre ambos países en una caída del PIB chino en un 1,3\%.

\subsection{PLAN MADE IN CHINA 2025: LA RELEVANCIA DEL COMERCIO ELECTRÓNICO}

En el presente estudio, se considera relevante incorporar como factor de análisis del desarrollo del Plan Made in China 2025, el comercio electrónico (Martínez-López, 2019). El siglo XXI es el siglo de la Economía Digital donde el protagonismo está en los avances tecnológicos como la analítica de volumen de información (Montoriol, 2015), la computación en nube, el internet de las cosas, la realidad virtual o el 5G (Rodríguez et al., 2020 y Sánchez-Cabrero et al., 2019).

Hay diferentes investigaciones que relacionan la innovación de las empresas chinas con su capacidad de adaptación al comercio electrónico (Wang y Wang, 2019). Li (2015) señaló que las empresas más tradicionales han tenido que cambiar su cadena de suministro para poder hacer frente a los retos que les suponía el comercio en línea. Shao y $\mathrm{Hu}$ 
(2016) analizaron, a través del caso de Alibaba, el impacto de la adopción del comercio electrónico y el desarrollo de una plataforma comercial en línea en la organización de la propia empresa.

El estudio de Wang y Wang (2019) resume que el comercio electrónico afecta principalmente en tres campos de la innovación: organización interna, la logística y las formas de comunicación. A nivel de organización interna el comercio en línea impacta sobre el marketing obligando al desarrollo de estrategias específicas para el entorno digital tanto de promoción como de gestión de clientes. En relación con la logística, el comercio electrónico estimula la optimización de la cadena de suministro de la industria. Por ejemplo, el manejo de un gran volumen de datos y su análisis favorece la competitividad y la eficiencia de la industria (Wright et al., 2019). Esta analítica de datos derivados de las transacciones en línea da una valiosa información de la respuesta tanto de proveedores como de clientes lo que ayuda a las empresas a la toma de decisiones más ajustadas al mercado. Por último, la mejora en el intercambio de información económica provoca un entorno económico que favorece la innovación corporativa como rasgo identitario.

El desarrollo del comercio electrónico en China ha supuesto que las empresas adopten una serie de avances tecnológicos necesarios para su éxito destacando entre ellas Tencent, Alibaba o Xiaomi. Las dos primeras con productos directamente relacionados con el comercio en línea y la última como fabricante del principal soporte usado en China para realizar las compras en este canal, los teléfonos inteligentes. El 98,6\% de los usuarios chinos de Internet lo realizan a través de estos dispositivos (ICEX, 2020).

Según Martínez-López y Jaén (2018) “el comercio electrónico es uno de los elementos fundamentales en la economía y una de las causas que han ayudado a la globalización económica”. Un elemento cada vez más presente, en 2019, según las estimaciones de la Unión Internacional de Telecomunicaciones, 4.536 millones de personas usaron Internet, lo que representa un 58,8\% del total de la población mundial y de éstos 2.068 millones utilizaron algún canal digital para comprar en línea (Emarketer, 2019).

En 2019, China lideraba el ranking de países con mayor número de usuarios de Internet alcanzando los 861 millones lo que suponía el 60\% de su población. Si lo comparamos con datos del año 2002 cuando tenían 22 millones de usuarios, han tenido un crecimiento del 
$3727,7 \%$. En relación con el grado de penetración, el 60\% es un porcentaje aún bajo con respecto al que tienen otros países como pueden ser Estados Unidos, Japón o Alemania que están por encima del 90\% (véase Tabla 2).

Tabla 2. Top 10 países con el mayor número de usuarios a nivel mundial en $2000-2019$.

\begin{tabular}{|c|c|c|c|c|c|c|}
\hline Top & País & $\begin{array}{c}\text { Población } \\
\text { total 2019 }\end{array}$ & $\begin{array}{c}\text { Usuarios } \\
\text { Internet 2019 }\end{array}$ & $\begin{array}{c}\text { Peso sobre } \\
\text { población 2019 }\end{array}$ & $\begin{array}{c}\text { Usuarios } \\
\text { Internet 2000 }\end{array}$ & $\begin{array}{c}\text { Aumento } \\
\text { desde 2002 }\end{array}$ \\
\hline 1 & China & 1.428 .194 .807 & 861.236 .259 & $60 \%$ & 22.500 .000 & $3727,7 \%$ \\
\hline 2 & India & 1.368 .737 .513 & 560.000 .000 & $41 \%$ & 5.000 .000 & $11100 \%$ \\
\hline 3 & EE. UU. & 329.093 .110 & 292.892 .868 & $89 \%$ & 95.354 .000 & $207,2 \%$ \\
\hline 4 & Brasil & 212.392 .717 & 149.057 .635 & $70 \%$ & 5.000 .000 & $2881,1 \%$ \\
\hline 5 & Indonesia & 269.536 .485 & 143.260 .000 & $53 \%$ & 2.000 .000 & $7063,0 \%$ \\
\hline 6 & Japón & 126.854 .745 & 118.626 .672 & $94 \%$ & 47.080 .000 & $151,9 \%$ \\
\hline 7 & Rusia & 143.895 .551 & 109.552 .842 & $76 \%$ & 3.100 .000 & $3433,9 \%$ \\
\hline 8 & México & 132.328 .035 & 88.000 .000 & $67 \%$ & 2.712 .400 & $3144,3 \%$ \\
\hline $\mathbf{9}$ & Alemania & 82.438 .639 & 79.127 .551 & $96 \%$ & 24.000 .000 & $229,7 \%$ \\
\hline 10 & Turquía & 82.961 .805 & 69.107 .183 & $83 \%$ & 2.000 .000 & $3355,3 \%$ \\
\hline
\end{tabular}

Fuente: Adaptado ${ }^{1}$ de Internet World Stats, 2019. Copyright 2020.

El grado de penetración indica una magnitud del intervalo de crecimiento que aún tiene China en relación con usuarios de Internet y por ende a potenciales consumidores en línea. De todas formas, ha sido un éxito que se haya alcanzado este volumen de usuarios que viene principalmente explicado por dotar al país de las infraestructuras necesarias a nivel de telecomunicaciones para poder acceder a Internet a través de los teléfonos inteligentes (veáse Figura 3).

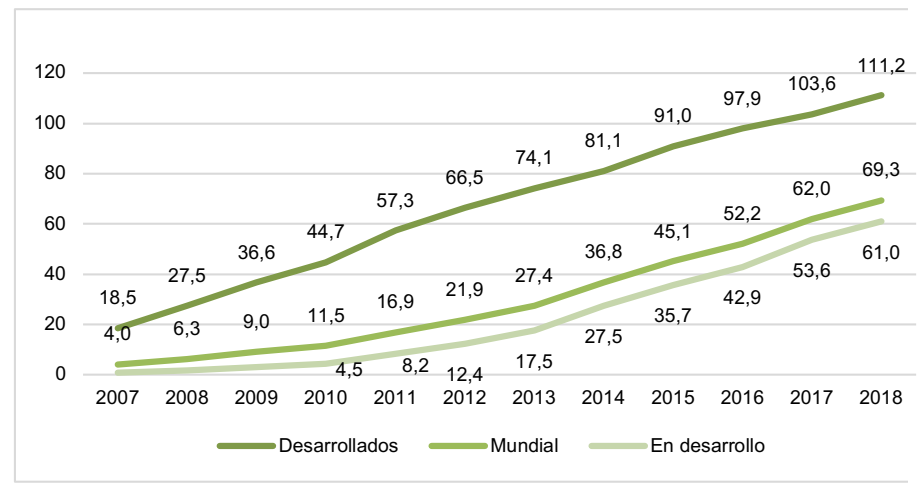

Figura 3. Porcentaje de dispositivos móviles con acceso a Internet por nivel de desarrollo, 2002-2018. Fuente: Adaptado de la Unión Internacional de Telecomunicaciones, 2019. 
Este crecimiento de los usuarios de Internet en Asia, gracias al acceso a través de teléfonos inteligentes, no solo ha sucedido en China, también destaca la India que alcanzó en 2019 los 560 millones o Indonesia con 143 millones (véase Tabla 2).

China también lideró el ranking de países con mayor volumen de venta a través de comercio electrónico en 2019 alcanzando los 1934 billones de dólares. Esto supuso un crecimiento respecto a 2019 del 27,3\% y un 55\% del peso sobre las ventas totales de las ventas en línea en todo el mundo. Los datos sobre comercio electrónico revelan que Asia está en el foco de su crecimiento con cuatro países en el top diez de usuarios y cinco en el de volumen de ventas (China, India, Japón, Corea del Sur e Indonesia, véase Tabla 2 y Tabla 3).

Tabla 3. Top 10 países con mayor volumen de ventas en línea 2018 - 2019 (en billones de dólares).

\begin{tabular}{|c|c|c|c|c|}
\hline Países & $\mathbf{2 0 1 8}$ & $\mathbf{2 0 1 9}$ & Crecimiento & $\begin{array}{c}\text { Peso Ventas } \\
\text { Totales }\end{array}$ \\
\hline China & 1520,1 & 1934,7 & $27,3 \%$ & $55 \%$ \\
\hline EE. UU. & 514,8 & 586,9 & $14,0 \%$ & $17 \%$ \\
\hline Reino Unido & 127,9 & 141,9 & $10,9 \%$ & $4 \%$ \\
\hline Japón & 110,9 & 115,4 & $4,0 \%$ & $3 \%$ \\
\hline Corea del Sur & 87,6 & 103,4 & $18,1 \%$ & $3 \%$ \\
\hline Alemania & 75,9 & 81,8 & $7,8 \%$ & $2 \%$ \\
\hline Francia & 62,2 & 69,4 & $11,5 \%$ & $2 \%$ \\
\hline Canadá & 41,1 & 49,8 & $21,1 \%$ & $1 \%$ \\
\hline India & 34,9 & 46,1 & $31,9 \%$ & $1 \%$ \\
\hline Rusia & 22,6 & 26,9 & $18,7 \%$ & $1 \%$ \\
\hline
\end{tabular}

Fuente: elaboración propia con datos de Emarketer (2019), y Statista (2019).

La innovación en las empresas chinas relacionadas con el comercio electrónico es una evidencia. El éxito de la conectividad en China gracias al Plan Made in China 2025 es una realidad. El estímulo a los principales operadores móviles (China Mobile, China Telecomo o China Unicom) es constante lo que provoca que la infraestructura digital mejore sensiblemente. Ejemplo de ello es el desarrollo de la tecnología 5G donde China tiene el mayor número de patentes del mundo.

La exigencia de los consumidores en línea chinos también estimula esa necesidad de innovación donde se necesita garantizar un elevado grado de reposición, de entrega y atención al cliente. La importancia de este tipo de cliente en el panorama de las ventas 
detallistas es fundamental ya que en 2019 el 36\% del total de éstas se hicieron a este cliente y se estima que en 2023 supongan el 64\% del total (Emarketer, 2019).

Se debe tener muy en cuenta que este volumen de clientes supone que China sea el principal operador de logística en línea suponiendo el $40 \%$ del total del servicio de entregas urgentes (ICEX, 2020).

Las innovaciones en el entorno online chino son muy diversas y pioneras en relación con el resto del mundo. Han desarrollado aplicaciones que cuentan con funciones y servicios bajo una misma plataforma generando super aplicaciones. En éstas el consumidor realiza todo desde dentro, busca la información, compra y paga desde el mismo sitio. Empresas como Alibaba y Tencent se han posicionado como referentes no solo como plataformas de comercio electrónico o redes sociales, sino por sus sistemas de pago y formas de financiación. Ejemplo de esto es la aplicación WeChat, de Tencent, una red social desde la que se pueden realizar compras, pedir un taxi, elegir la comida, jugar a algún videojuego, escuchar música o usarla como plataforma de pago. Con esta estrategia han conseguido que el usuario chino permanezca entre cuatro y cinco horas al día en la misma aplicación (Emarketservices, 2020).

La innovación financiera que han desarrollado estas empresas a través de sus plataformas ha supuesto que las aplicaciones de los bancos hayan perdido peso y los consumidores locales hayan adoptado WeChat Pay o la versión desarrollada por Alibaba, Alipay.

\section{El impacto del GOVID-19 en el comercio electrónico en China}

China ha experimentado una paralización económica generalizada debido a la enfermedad por coronavirus 2019 (COVID-19) como se indicó en puntos anteriores.

El impacto del COVID-19 en el comercio minorista ya se ha reflejado parcialmente en la fuerte caída en el crecimiento de las ventas minoristas para el período de enero y febrero, que generalmente es un período de auge para el consumo. Las ventas minoristas totales de bienes de consumo cayeron un 20,5\% interanual nominalmente en los primeros dos meses del año, mientras que las ventas de catering cayeron un 43,1\% interanual. La mayoría de las categorías de productos registraron una caída significativa en las ventas, excepto los 
cereales, el aceite, los productos alimenticios, las bebidas y los medicamentos (National Bureau of Statistics of China, 2020).

Sin embargo, esta pandemia ha traído nuevas oportunidades en el comercio electrónico, ya que tanto marcas, como detallistas sin presencia online, han buscado la forma de potenciar este canal de venta.

Las categorías de productos que más se consumían en China antes de la situación provocada por el COVID-19 eran Moda y Belleza, Electrónica o Juegos (véase Tabla 4). Aparece en quinto lugar la alimentación y cuidado personal (Hootsuite, 2019).

Tabla 4. Principales categorías más compradas en China ordenadas por volumen de ventas (billones de dólares).

\begin{tabular}{|r|c|c|}
\hline Categorías & Ventas & Crecimiento \\
\hline Moda y belleza & 164,2 & $26 \%$ \\
\hline Electrónica & 112,7 & $19 \%$ \\
\hline Juegos, manualidades y pasatiempos & 97,8 & $28 \%$ \\
\hline Muebles y electrodomésticos & 80,8 & $17 \%$ \\
\hline Alimentación y cuidado personal & 43,4 & $34 \%$ \\
\hline Viajes & 27,3 & $23 \%$ \\
\hline Videojuegos & 11,7 & $10 \%$ \\
\hline Música Digital & 0,5 & $26 \%$ \\
\hline
\end{tabular}

Fuente: elaboración propia con datos de Hootsuite (2019).

En la primera mitad de este año esto ha cambiado. Las ventas en línea continuaron creciendo rápidamente en comparación con las ventas físicas. En enero-mayo de 2020, las ventas minoristas en línea aumentaron un 4,5\% interanual. En particular, las ventas minoristas en línea de alimentos y productos de uso diario aumentaron en un $37.0 \%$ y $14.9 \%$ interanual respectivamente, lo que demuestra que los consumidores aumentaron su gasto en línea en estas categorías en medio de los requisitos de distanciamiento social y bloqueo (NBS, 2020).

Este hecho va a ser muy significativo en el futuro del comercio electrónico en China, ya que era la última barrera que faltaba por superar claramente (Martínez-López, 2019): ahora el consumidor en línea chino es capaz de organizar sus compras de productos de uso diario y productos frescos a través de la compra online.

El COVID-19 ha estimulado al comercio electrónico en China principalmente en cuatro líneas (Fung Business Intelligence, 2020): 
1. Las principales empresas de comercio electrónico como Alibaba Group, JD o Pinduoduo han dado soporte tanto a marcas como a minoristas para ayudar a los comerciantes a superar la crisis. Han dado apoyo financiero, logístico y de marketing. Esto ha supuesto un estímulo a la innovación de muchas organizaciones que no tenían presencia online.

2. La herramienta clave a nivel de ventas y marketing han sido las transmisiones en vivo para mostrar a los consumidores el uso de los diferentes productos y servicios.

3. A nivel logístico se han desarrollado diferentes formas de entregas sin contacto. Se han usado vehículos sin conductor o drones. Así ha sido el caso de la empresa Meituan Maidai con sus vehículos autónomos o JD con las entregas con sus drones.

4. El comercio electrónico a través de las redes sociales gana fuerza. Herramientas como WeChat Mini Programs no han servido solo a particulares y micro-empresas a promocionar y poner al alcance de los consumidores sus productos sino que también ha sido una opción elegida durante esta etapa por los grandes minoristas. Se han sumado a esta estrategia, por ejemplo, empresas de ropa como Dazzle oJNBY.

5. La demanda de productos de consumo frecuente como alimentos frescos, comestibles y otras necesidades diarias ha crecido significativamente como indican las principales plataformas electrónicas de estos productos JD Daojia, Yonghui Daojia y Dmall. Durante esta etapa han mejorado la facilidad para realizar los pedidos y la velocidad de entrega de los pedidos.

Se puede concluir que el comercio electrónico en China es fuente constante de innovación y de estímulo para las empresas que operan en él, sirviendo de apoyo y ayuda a aquellas organizaciones que aún no operaban en él para superar la pandemia ocasionada por el COVID-19.

\section{CONCLUSIONES}

A lo largo de este trabajo se ha realizado un análisis del proceso de desarrollo que ha experimentado China desde que se pusieron en marcha las medidas de la estrategia económica del actual presidente Xi Jinping a finales del año 2013. 
Se ha hecho una revisión de tres de los principales retos a los que se está enfrentando el país del dragón: eliminación de la pobreza, lucha por equiparación del desarrollo regional y transformación del modelo productivo hacia una producción de mayor valor añadido con más componente de innovación. Dentro de este último eje, se ha profundizado en el análisis de la evolución del comercio electrónico.

Se ha tenido en cuenta los efectos de la pandemia causada por el COVID-19 en esta senda de transformaciones. Los efectos del COVID-19 tienen consecuencias en el desarrollo económico corto plazo: El objetivo de erradicar la pobreza rural en el año 2020 va a verse demorado, parte de la inversión orientada al desarrollo de infraestructuras para equilibrar el equilibrio regional va a desviarse al sistema socio sanitario y

En el capítulo tecnológico, la pandemia ha supuesto una estimulación para el desarrollo de la innovación (aplicaciones de inteligencia artificial, robótica) con especial reflejo en el comercio electrónico donde empresas que antes no operaban a través de este canal ahora lo están haciendo con el apoyo y ayuda de aquellas que ya estaban haciéndolo. Y estas han puesto en práctica innovaciones en desarrollo como entrega de paquetería a través de vehículos autónomos.

Es dificil hacer proyecciones en el corto plazo considerando la gran incertidumbre que caracteriza al panorama internacional. El Fondo Monetario Internacional (2020) pone de manifiesto la irregular incidencia de la pandemia COVID-19 a lo largo de las diferentes regiones del mundo: prevé que Asia va a ser el continente que más va a crecer en 2021: Estima que su PIB crecerá un 8,5\% frente al escaso 4,7\% que se estima para Europa y al 3,4\% para América Latina.

Si proyectamos la mirada hacia el futuro, más allá de las turbulencias del corto plazo propias del ciclo actual, podemos confirmar que en el S XXI China será la principal economía del planeta y Asia será el protagonista desde una perspectiva económica y social. Según las proyecciones de la OCDE (2019), reflejadas en la Figura 4, se puede observar que China va a consolidar su posición de líder en términos de PIB y que India superará a los países de la zona euro entre 2025 u 2030 y a los Estados Unidos entre 2035 y 2040. Por tanto, dentro de aproximadamente 20 años, las dos mayores potencias económicas del mundo estarán en Asia, muy por encima de las potencias occidentales. 
Proyección a largo plazo del PIB por país entre 2020 y 2060

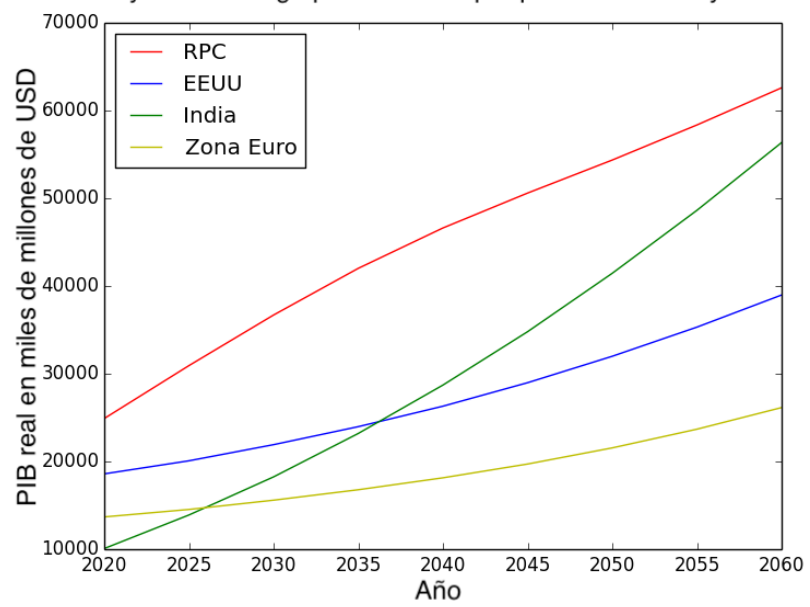

Figura 4. Previsiones a largo plazo de PIB por países entre 2020 y 2060 (en millones de dólares). Fuente: elaboración propia a partir de datos de la OCDE (2019).

Nota: los datos aparecen expresados en términos constantes con ppp de 2010.

Por todo ello, se consideran alcanzados tanto el objetivo principal como los secundarios del presente estudio. La principal limitación de esta investigación, si bien se ha profundizado en un factor clave como es el comercio electrónico, ha sido no incluir más factores importantes en el desarrollo del Plan Made in China 2025 como puede ser concretar el plan de infraestructuras tecnológicas 5G. De esta limitación se desprende para futuras líneas de investigación, la relevancia de la creación de un barómetro que incluya tanto las variables aquí tratadas como otras con el fin de poder dotar de un mayor conocimiento de China tanto a la comunidad científica como a la empresarial. De esta forma, la comunidad podrá seguir profundizando en el desarrollo de Asia, mientras que las empresas dispondrían de una herramienta para la toma de decisiones.

\section{REFERENCIAS BIBLIOGRÁFICAS}

Asia Society Policy Institute y The Rhodium Group. (2020). China Dashboard Spring 2020 Update. https://bit.ly/3384T29

Banco Mundial. (2020). Poverty Data and equity data portal. http:// povertydata.worldbank. $\mathrm{org} /$ poverty/country/CHN 
Blanco, A. (2019). China como líder innovador, entre el éxito y las dudas. Análisis del Real Instituto Elcano, 75. https://bit.ly/3hPdKd7

Boo, G., David, M., y Simpfendorfer, B. (2020). How will COVID-19 affect China's Belt and Road Initiative? https://bit.ly/2EzWjiA

Bregolat, E. (2007). La Segunda Revolución China. Destino.

Bregolat, E. (2018). Made in China 2025. Estudios de Política Exterior, 185, 22-27.

Cámara de Comercio Europea en Ghina. (2018). European Business in China Position Paper, 2018/2019. https://bit.ly/39H3hOn

Deloitte Insights. (2018). Embracing the BRI Ecosystem in 2018. https://bit.ly/2ErrqN4

Emarketer. (2019). China Ecommerce 2019. Retail Ecommerce Stays Strong as Geopolitical and Economic Headwinds Dampen Overall Retail SectoR. Emarketer.

Emarketer. (2019). Global Ecommerce 2019. Emarketer.

Emarketservices. (2020). Entorno digital y redes sociales en China 2020. ICEX.

Fanjul, E. (2018, 15 de noviembre). China: 40 años de la mayor revolución económica de la historia. Elcano Blog. https://bit.ly/2P8rvrb

Fondo Monetario Internacional. (2020). World Economic Outlook. Abril, 2020: The Great Lockdown. https://bit.ly/3gelAMZ

Fung Business Intelligence. (2020). China Retail \& E-commerce Quarterly. FBI.

Hootsuite. (2019). Digital 2019. We Are Social Ltd.

ICEX. (2020). Informe e-País: El comercio electrónico en China. ICEX.

Li, H. (2015). Research on the innovation and development of traditional industry under the background of e-commerce. Macroeconomics Managament, 2, 79-82.

Lieberthal, K. (2011). Managing the China Challenge: How to achieve corporate success in the People's Republic. Brookings Institution Press. 
Martínez, F., y Jaén, R. (2018). Análisis evolutivo y dimensional del concepto de comercio electrónico. Saberes, 1. https://revistas.uax.es/index.php/sab/article/ view/1265/1029

Martínez-López, F. (2019). Enfoque estratégico para la toma de decisiones en el comercio electrónico en Asia Oriental [conferencia número 14]. Europa y Asia: un destino común, Programa de Conferencias Fundación Alfonso X el Sabio - One Asia, Villanueva de la Cañada, Madrid, España.

Montoriol, J. (2015). Digitalizarse o morir: la transformación digital de industrias y empresas. Dossier: La economía digital. La Caixa Research.

National Bureau of Statistics of China. (2020). Total Retail Sales of Consumer Goods. NBS.

OECD. (2019). Real GDP long-term forecast (indicator). https://data.oecd.org/gdp/real-gdplong-term-forecast.htm

Rodríguez, L.F., Perona, M., Martínez-López, F., Reichardt, S., Fuente, M., y Martínez-López, L. (2020). III Barómetro DCH sobre la Digitalización de la función de RR.HH. en España (III). Capital Humano, marzo, 86-91.

Ryder, H. (2017, 28 de marzo). The end of Poverty in China? Project Syndicate. https://bit. ly/39Emtfm

Sartori, G., y Morlino, L. (1994). La comparación en las ciencias sociales. Alianza Editorial.

Shao, P., y Hu, P. (2016). Case study on business model innovation and evolution of e-commerce platform. Science Research Management, 7, 81-88.

Statista. (2019). Ventas mundiales comercio electrónico 2011-2020. Statista Research Department.

Textor, G. (2020). Per capita gross domestic product (GDP) in China 2018, by province. Statista Research Department. 
Wang, X., y Wang, H. A. (2019). Study on Sustaining Corporate Innovation with E-Commerce in China. Sustainability, 11(23), Article 6604. https://doi.org/10.3390/ sul1236604

Wolff, R., y Resnick, S. (2012). Contending Economic Theories: Neoclassical, Keynesian, and Marxian. The MIT Press.

Wright, L.T., Robin, R., Stone, M., y Aravopoulou, D.E. (2019). Adoption of big data technology for innovation in B2B marketing. Fournal of Business-to-Business Marketing, 34, 281-293. https://doi.org/10.1080/1051712X.2019.1611082 